\title{
Colorectal Signet Ring Cell Carcinoma in a Young Pregnant Woman
}

\author{
Francisco Ibargüengoitia Ochoa ${ }^{a}$ Gerardo Miranda Dévora ${ }^{b}$ \\ Leonardo Silva Lino ${ }^{c}$ Cintia Sepulveda Rivera ${ }^{a}$ Diego González Vázquez ${ }^{a}$ \\ Milagros Pérez Quintanillad \\ ${ }^{a}$ Gynecology and Obstetrics Department, Instituto Nacional de Perinatología, Mexico \\ City, Mexico; ${ }^{b}$ Gastroenterology Department, Instituto Nacional de Cancerología, Mexico \\ City, Mexico; 'Pathology Service, Instituto Nacional de Cancerología, Mexico City, Mexico; \\ dGynecology Oncology Department, Instituto Nacional de Perinatología, Mexico City, \\ Mexico
}

\author{
Keywords \\ Colorectal cancer $\cdot$ Signet ring cell carcinoma $\cdot$ Pregnancy $\cdot$ Mortality
}

\section{Abstract}

Colorectal cancer during pregnancy is one of the less common neoplasms with an incidence of 0.8 in 100,000 pregnancies. Primary colonic signet ring cell carcinoma is a weird variety, characterized by a poor histologic differentiation, with a high morbidity-mortality rate. The case of a 24-year-old patient with a 22-week-old pregnancy and colorectal cancer stage IV in palliative state is presented, with a devastating result. Early diagnosis represents a challenge because of the presentation form and the histologic aggressiveness of this disease. We suggest that colorectal cancer during pregnancy must be treated by a multidisciplinary team.

\section{Introduction}

Colorectal cancer during pregnancy is one of the less common neoplasms with an incidence of 0.8 in 100,000 pregnancies [1]. A compilation of 4 studies, containing approximately 18 million people, found pregnancy-associated colorectal cancer in $0.0022 \%$ [2]. Diagnosis and therapeutic management of pregnant patients with colorectal cancer are especially difficult because the symptoms are easily attributable to the pregnancy; so that most frequently, patients present at an advanced stage based on a late diagnosis [3]. 


\section{Case Reports in \\ Oncology}

\begin{tabular}{l|l}
\hline Case Rep Oncol 2020;13:182-187 \\
\hline DOI: 10.1159/000504472 & $\begin{array}{l}\text { ○ 2020 The Author(s). Published by S. Karger AG, Basel } \\
\text { www.karger.com/cro }\end{array}$ \\
\hline
\end{tabular}

Ibargüengoitia Ochoa et al.: Colorectal Signet Ring Cell Carcinoma in a Young Pregnant Woman
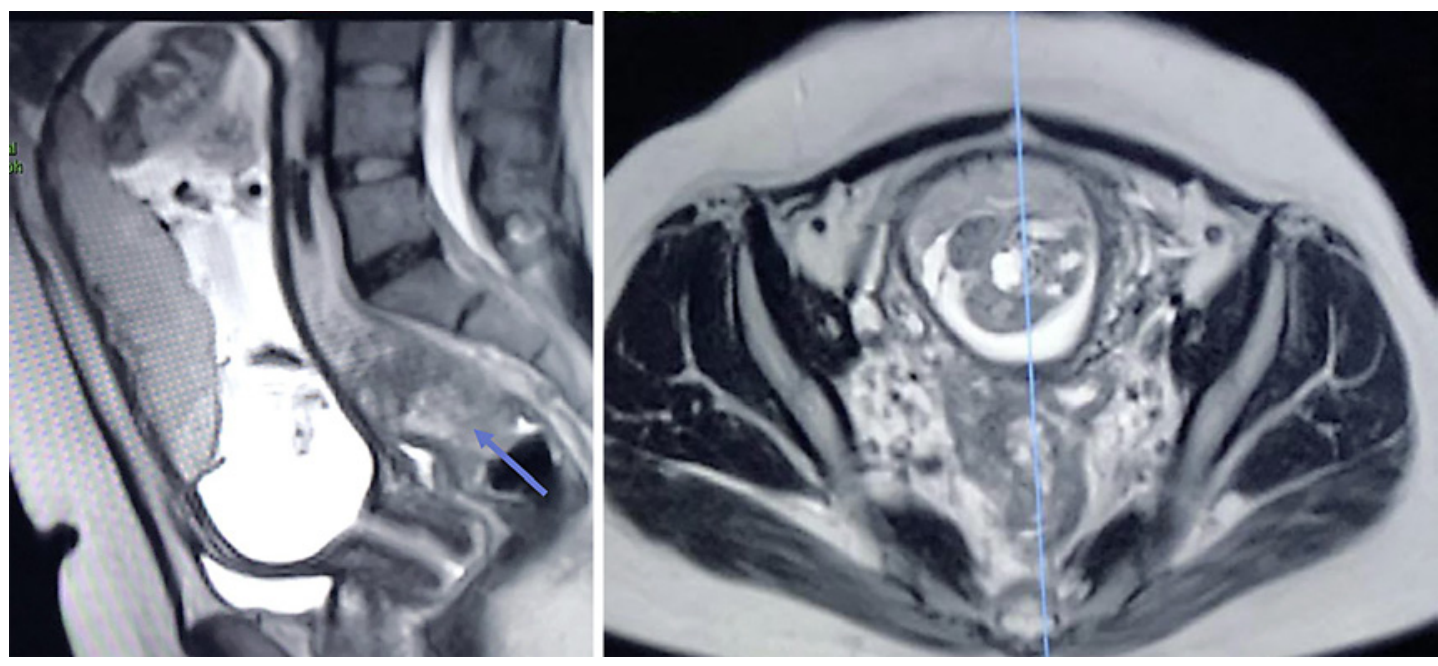

Fig. 1. MRI of the pelvis, sagittal and transversal image. The tumor is located in the rectum, occluding $90 \%$ of its light (blue line and arrow).

Fig. 2. Coronal MRI of the pelvis showing a living 22-week-old fetus.

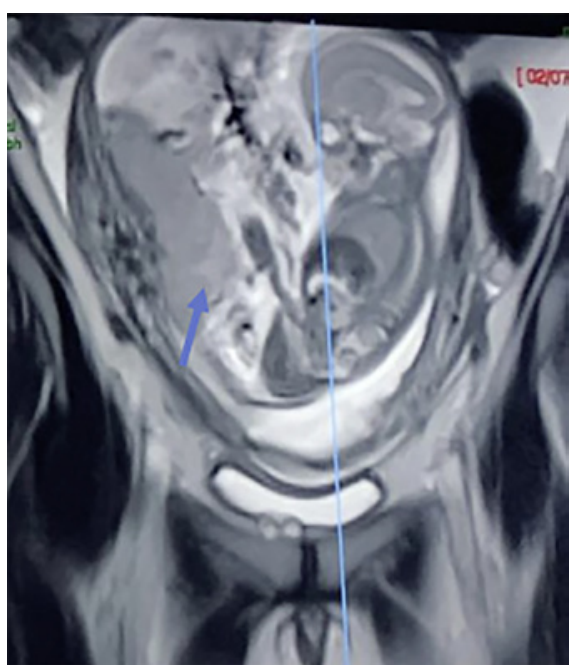

There are only limited series reports and, to date, there are no guidelines or consensus available about colorectal management during pregnancy $[2,4,5]$. Primary colonic signet ring cell carcinoma is a weird type that is characterized by a poor histologic differentiation, whose cells contain in a characteristic way a typical mucin morphology which pushes the nucleus to the periphery resulting in the typical signet ring morphology [6]. We describe a pregnant patient with colonic signet ring cell carcinoma.

\section{Case Report}

Our patient was a 24-year-old pregnant female (gravida: 2, para: 1), born and resident in a rural area, with a family history of cancer: maternal grandmother with thyroid carcinoma and grandfather with prostate carcinoma. Alcoholism and smoking were denied. Antecedent was a living birth 4 years ago. She was referred to our institution with a 22 -week-old normal pregnancy. She was referred for occasional nausea and vomiting, constipation, and 1-month 
Fig. 3. Under magnification, colonic epithelium infiltrates by mucin lakes and signet ring cells.

Ibargüengoitia Ochoa et al.: Colorectal Signet Ring Cell Carcinoma in a Young Pregnant Woman

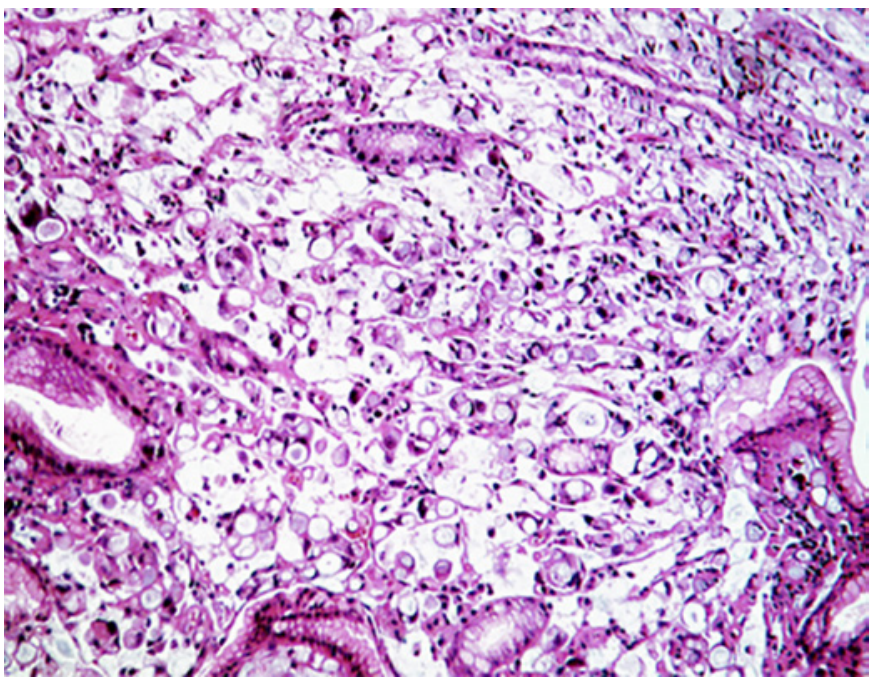

Fig. 4. Under magnification, ring cells are observed, without large cytoplasm, eccentric with cytoplasmic vacuoles and hyperchromatic nuclei, peripheral displacement.

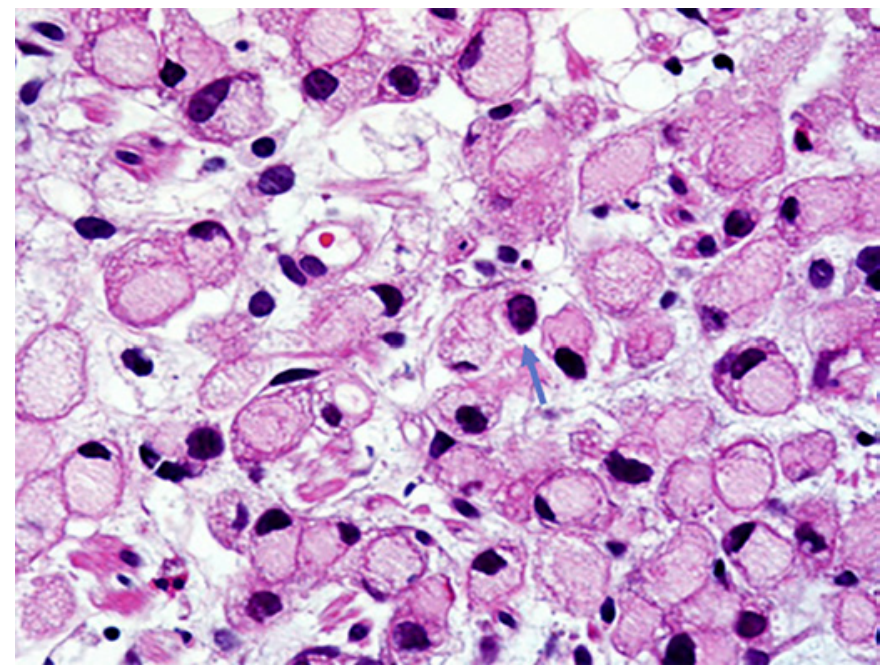

dyspnea, as well as a 10-kg weight loss in the last 4 months; with loss of functional capacity. Laboratory exams highlighted $\mathrm{Hb} 9.9 \mathrm{~g} / \mathrm{dL}$, platelets 553,000/ $\mu \mathrm{L}$, CA125 172 IU (normal: 0-32), AFP 76 IU (normal: 0-20), CEA 29 IU (normal: 0-3.4), and CA19-9 2,602 IU (normal: $0-39$ ). Abdominal ultrasound showed ascitic fluid, suggestive of mesenteric thickening carcinomatosis.

Magnetic resonance imaging (MRI) showed a semioval irregular shape, next to the rectal ampule measuring $2.5 \times 2.6 \times 2.6 \mathrm{~cm}$ (Fig. 1 and 2). Colonoscopy was performed finding a rectal tumor covering $90 \%$ of the colon. A biopsy was performed with the histopathologic result of colonic signet ring cell carcinoma (Fig. 3 and 4). Patient was evaluated by a multidisciplinary team from the National Cancer Institute, Mexico, including surgical oncology, medical oncology, and gynecologic oncology. The conclusion was rectal carcinoma clinical stage IV and - given the clinical condition and advanced stage of the disease - palliative treatment is required.

Pregnancy was terminated by vaginal delivery 3 weeks after diagnosis, obtaining a 742-g newborn at 28 weeks of gestation, who died 15 days after. Three weeks after delivery, the patient presented signs of intestinal occlusion, putting on a palliative stent. Our patient died 25 days after having given birth. 
Ibargüengoitia Ochoa et al.: Colorectal Signet Ring Cell Carcinoma in a Young Pregnant Woman

\section{Discussion}

The incidence of colorectal cancer is increasing in people $<50$ years old [7]. Only $21 \%$ of all patients diagnosed with colorectal cancer are diagnosed before 55 years of age [7].

Early diagnosis of colorectal cancer during pregnancy is difficult because the disease does not present specific symptoms and the presenting symptoms are associated with normal physiologic changes during a pregnancy; until an obstruction or perforation happens. The clinical presentation includes fatigue, anemia, constipation, pain, and rectal hemorrhage. Weight loss with or without other symptoms must not be underestimated in pregnant women [5].

During pregnancy, MRI is the chosen imaging study [2]. Colonoscopy can be performed safely during gestation. In this case, our patient presented with an abrupt growth of her abdominal perimeter not linked to pregnancy weeks, a pelvic MRI was performed to discard an adnexal tumor, finding ascites, carcinomatosis, and a rectal ampule tumor. The differential diagnosis was epithelial ovarian cancer because of the clinical presentation of the patient.

In the literature, there are two series that review colorectal cancer associated with pregnancy. The most relevant was published by Pellino et al. [2] in 2017. This is a recompilation of 79 papers that includes 119 patients with an average age at neoplasm diagnosis of 32 years (range: 17-46). Diagnosis was made during the second and third trimester in 41 and 47\%, respectively. Localization of the tumor was the colon in 53\% and the rectum in $44 \%$. The average survival rate was 36 months (range: 0-360); the lowest survival rate was diagnosed in the second trimester (30 months) [2]. The presented case is a young woman in her third decade of life, with an atypical clinical presentation of colorectal cancer, with an uncommon histologic lineage, that ended with perinatal and maternal death.

The second series is from Kocián et al. [5] published in 2019. The authors compiled data from the International Network on Cancer, Infertility and Pregnancy which collects oncologic, obstetric, and perinatal information related to cancer diagnosed in pregnant women. The series included 41 patients (27 patients presented with colon cancer, 14 with rectal cancer). Mean age at diagnosis was 32 years (range: 24-34). Multiparity was noted in 69\%, non-acute clinical presentation in 75\% (constipation in 4.9\%, weigh loss in $2.4 \%$ ). A clinical diagnosis was made in the second trimester in $46 \%$, by imaging in $9.8 \%$ and endoscopy in $65.9 \%$. The tumor was located in the colon in 51\%. 41.5\% were at clinical stage IV. $78 \%$ of the cases obtained a pre-term newborn with an average gestational age of 35 weeks (range: 27-40) [5]. The patients in the second trimester seem to have a poor life prognosis; as in our case.

Primary colonic signet ring cell carcinoma was first described in 1951 [8]. The estimated incidence is $0.1-2.4 \%$. A large population study that included 196,757 colorectal cancer cases, of which $45 \%$ were women, showed that $2.7 \%$ of the patients were $<45$ years old [8]. Colonic signet ring cell carcinoma is known to be a very aggressive colonic cancer form [6].

Late clinical presentation is common and implies an important delay in diagnosis; finding of this neoplasm is by itself a bad prognostic factor, independent of comorbidities and tumoral extension. Aggressiveness with diffuse intratumoral infiltration and rapid extension by lymphatic and peritoneal continuity is common. The most common presentation is at stages III and IV with a low survival rate. A review was conducted at the National Cancer Institute, Mexico, a national reference center, finding 10 signet ring cell adenocarcinomas in 12 years; this shows the histopathologic challenge for diagnosis.

In a review of 776 patients, women under the age of 35 years had a worse prognosis [2]. Ovarian metastasis was present in $23 \%$ of all pregnant women with colorectal cancer [2]. Metastatic colorectal cancer is a serious matter to be taken into account because of the extension of peritoneal invasion in these cases. 
Colorectal cancer treatment in pregnancy is influenced by different factors including location of the tumor, clinical stage, surgical possibility (elective/urgent), and gestational age. If a diagnosis of colorectal cancer is made at an early gestational stage, surgical resection can be done without compromising the fetus. In a pregnancy with gestational age $<20$ weeks, an abortion can beadvisable. The patient may refuse it and accepts the risks of disease progression. Chemotherapy based on 5-fluorouracil, leucovorin, and oxaliplatin is feasible in colorectal cancer cases during pregnancy in the second and third trimester, although it is associated with growth delay in the fetus.

Vaginal birth can be mandatory depending on tumor location $[2,5]$. Colostomy or stent placement (as a less invasive palliative therapy) may be need if there is evidence of an obstruction [3].

Young age has not been identified as a clinical risk factor for colorectal cancer. The risk in women $<40$ years old can be 2-4 times higher in relation to the risk in their grandparents, suggesting that health habit changes and environmental factors have an impact on the risk of this neoplasm. In this group, the neoplasm tends to be diagnosed at later clinical stages and in a distal colon location [8].

Cancer during pregnancy is a devastating event that requires a multidisciplinary management. Every case must be considered individually.

\section{Conclusion}

Colorectal signet ring cell carcinoma and pregnancy is a strange entity with high death rates and perinatal mortality that must be taken into account. Early diagnosis is a challenge because of the presenting symptoms and the aggressiveness of histology, not only to general doctors, but also to gynecologists, pathologists, oncologists, and palliative care, therefore multidisciplinary management must be done.

\section{Acknowledgment}

The author thank the residents of gynecology and obstetrics of the Instituto Nacional de Perinatologia and the residents from Oncology Surgery of the Instituto Nacional de Cancerología in Mexico.

\section{Statement of Ethics}

Our case was reviewed by the Ethics Committee of the Instituto Nacional de Perinatologia. We have a signed consent for the publication of this case from our patient's spouse.

\section{Disclosure Statement}

There is no interest conflict by the authors.

\section{Funding Sources}

The authors did not receive any specific funding for this work. 
Ibargüengoitia Ochoa et al.: Colorectal Signet Ring Cell Carcinoma in a Young Pregnant Woman

\section{Author Contributions}

Francisco Ibargüengoitia Ochoa: conception, drafting, and research of the work. Gerardo Miranda Dévora: link with INCan (National Cancer Institute), Mexico, recollection of clinical data. Leonardo Silva Lino: images of pathology. Cintia Sepulveda Rivera: revisions of the manuscript. Diego González Vázquez: English translation. Milagros Pérez Quintanilla: conception and design of the word, critical input for important content, and administrative requirements.

\section{References}

1 Lee YY, Roberts CL, Dobbins T, Stavrou E, Black K, Morris J, et al. Incidence and outcomes of pregnancy associated cancer in Australia, 1994-2008: a population-based linkage study. BJOG. 2012;119:1572-82.

2 Pellino G, Simillis C, Kontovounsios C. Colorectal cancer diagnosed during pregnancy: systematic review and treatment pathways. Eur J Gastreoenterol Hepatol. 2017;29:743-53.

3 Capell MS. Colon cancer during pregnancy. Review. The gastroentherologist's perspective. Gastroenterol Clin North Am. 1998;27:225-56.

4 Mc Cormick A, Peterson E. Cancer in pregnancy. Rev Obstet Gynecol Clin N Am. 2018;45:187-200.

5 Kocián P, de Haan J, Cardowick EH, Uzan C, Lok CAR, Fruscio R, et al.; Writing Committee of the International Network on Cancer, Infertility and Pregnancy (INCIP) on this particular manuscript. Management and outcome of colorectal cancer during pregnancy: report of 41 cases. Acta Chir Belg. 2019;119(3):166-75.

6 Mora GI, Di Martino M, Muñoz de Nova J, Viamontes Ugalde FE, Rodríguez Sánchez A. Carcinoma primario de colon con células en anillo de sello: una rara entidad de mal pronóstico. Comunicación de 2 casos. Rev Gastroenterol Mex. 2017;83:358-60.

7 Siegel RL, Miller KD, Fedewa SA, Ahnen DJ, Meester RGS, Barzi A, et al. Colorectal cancer statistics, 2017. CA Cancer J Clin. 2017;67:177-93.

8 Huang B, Ni M, Chen C, Feng Y, Cai S. Younger age is associated with poorer survival in patients with signetring cell carcinoma of the colon without distant metastasis. Gastroenterol Res Pract. 2016;2016:2913493. 